\title{
Comparison of Morning and Evening/Night Dosing on the Efficacy of Escitalopram in Major Depressive Disorder at Naturalistic Setting
}

\author{
Kyu-Man Han and Ho-Kyoung Yoon \\ Department of Psychiatry, Korea University College of Medicine, Seoul, Korea
}

\begin{abstract}
Objective: Recent epidemiological and clinical studies have revealed that timing medications to the individual's biological clock could improve their effectiveness and reduce side effects. We aimed to investigate the association of escitalopram dosing time with treatment response and changes in global depressive and insomnia symptoms after 8-week of antidepressant treatment in a naturalistic setting in patients with major depressive disorder (MDD). Methods: A total of 71 patients with MDD taking escitalopram were recruited from the outpatient psychiatric clinic in a university hospital. The severity of depressive symptoms of all patients was assessed using the 17-item Hamilton Depression Rating Scale (HDRS) at the baseline and 8-week after the antidepressant treatment. The patients were classified into the Morning or Evening/Night group according to their time of escitalopram single dose. Results: The time of escitalopram single dose (morning vs. evening/night) was not associated with treatment response after 8-week of treatment when controlling for potential confounding factors in patients with MDD. No significant difference in changes in total HDRS score and insomnia-related subscale score was observed between the Morning and Evening/ Night groups. Conclusion: The present study suggests that the time of escitalopram single dose did not affect the treatment response or changes in global depressive and insomnia symptoms after 8-week of antidepressant treatment in a naturalistic setting.
\end{abstract}

Key Words: Chronotherapy major depressive disorder; Time of dose; Escitalopram; Treatment response; Insomnia

Received: November 15, 2019 Revised: December 10, 2019 Accepted: December 11, 2019

Corresponding author: Ho-Kyoung Yoon, MD, PhD, Department of Psychiatry, Korea University College of Medicine, 73 Goryeodae-ro, Seongbuk-gu, Seoul 02841, Korea. Tel: 82-31-412-5140, Fax: 82-31-412-5144, E-mail: hkhkgogo@korea.edu

(a) This is an Open Access article distributed under the terms of the Creative Commons Attribution Non-Commercial License (https://creativecommons.org/licenses/bync/4.0) which permits unrestricted non-commercial use, distribution, and reproduction in any medium, provided the original work is properly cited.

\section{INTRODUCTION}

A variety of epidemiological and genetic studies have revealed that disturbances of circadian rhythms can be directly linked to the risk of cancer development [1,2]. This is because cell cycle regulators, which are essential for cell proliferation and at the same time closely associated with tumor production, are under the control of the circadian clock. Interestingly, in the case of rapidly dividing cells, such as tumor cells or stem cells, the expression of the biological clock genes is significantly suppressed. This early study results have raised the possibility that the activation of the circadian biological clock plays a key role in tumor production and its treatment.

In particular, since the study of the biological clock was conducted in earnest, it has been known that the efficacy of anticancer drugs can be maximized and the side effects on normal cells can be minimized at the specific time of day. In experiments in mice, Lévi [3] has shown that the tolerability and effectiveness of chemotherapy varies at different times of the day and night. By using mathematical models he can predict the optimal time at which to give an animal a drug to maximise its effectiveness and tolerability. This concept of the timing administration of the anticancer drug is a representative example of chronotherapy that optimizes the therapeutic effect by using the inherent rhythm of human. In addition, cancer patients who had severe circadian rhythm disturbances showed a much better prognosis if treatment was combined with strategies to improve circadian rhythm such as strictly following the sleep-wake cycle during chemotherapy [4].

Investigators realized that cancer was not the only disease likely to be affected by circadian rhythm while chronotherapy was gaining recognition in the oncology research field. Clinical trials 
revealed that it is necessary to consider the application of chronotherapy in order to achieve better clinical outcomes with fewer side effects of prednisolone for the treatment of inflammation and auto-immune diseases [5]. Cardiovascular events were also recognized early on to cycle throughout the day, as early study showed that most patients admitted for heart attacks tended to experience their symptoms in the morning [6]. They suggested that if the rhythmic processes that drive the circadian rhythm of myocardial-infarction onset can be identified, their modification may delay or prevent the occurrence of infarction. Recent studies found that the rate-limiting enzyme regulating the synthesis of cholesterol in rats was most active at night, for example [7]. Subsequent showed cholesterol-lowering drugs such as statins are most effective when taken at bedtime [8]. Accordingly, the FDA has long recommended taking such medications in the evening.

In the past when antidepressant-trials were done, time-of-day information was often not considered. It is very likely that treatment responses have not meet the criteria not because they did not work or the mechanisms were wrong, but simply because time of administration was not taken into account. Therefore, in the present study, we aimed to investigate the association of escitalopram dosing time with treatment response after 8-week of antidepressant treatment in a naturalistic setting in patients with major depressive disorder (MDD). We also investigated the potential effects of escitalopram dosing time on the changes in global depressive symptoms and insomnia severity after 8-week of antidepressant treatment in patients with MDD.

\section{METHODS}

\section{Participants}

In the present study, a total of 71 patients with MDD were recruited from the outpatient psychiatric clinic of Korea University Anam Hospital located in Seoul, Republic of Korea. As a naturalistic setting, the inclusion criteria were patients with MDD ranging from 18 to 65 years and taking escitalopram $(5-30 \mathrm{mg})$ for 8-week antidepressant treatment. At baseline, two board-certified psychiatrists made a diagnosis of MDD using a structured clinical interview based on the Diagnostic and Statistical Manual of Mental Disorders, fourth edition, text revision Axis I disorders. The following exclusion criteria were applied: patients with 1) comorbid other major psychiatric disorders, 2) current psychotic symptoms, 3) high suicidal risk requiring inpatient treatment, 4) history of a unstable medical illness, 5) primary neurological disorders (e.g., Parkinson's disease, cerebrovascular disease, epilepsy), 6) abnormal results on physical examination or laboratory tests, or 7) pregnancy. The protocols of this study were approved by the Institutional Review Board of Korea University Anam Hospital (No. 2016AN0213). All subjects gave written informed consent for their participation for the study with full understanding. All methods in the present study were performed in accordance with the approved guidelines and Declaration of Helsinki.

\section{Group classification and medication}

The patients were classified to the morning or evening/night group according to their morning or evening/night single dose of escitalopram (ranged from 5-30 mg) at the baseline. The patients in the morning group took their escitalopram 30 minutes after their breakfast, while those in the evening/night group took their escitalopram 30 minutes after their dinner, at $9 \mathrm{PM}$, or before sleep at night. We confirmed that the dosage and the time of dose of escitalopram did not change during 8-week of antidepressant treatment in all patients. The combination or augmentation treatment during the 8 -week of the treatment were as follows ( $\mathrm{n}=20$ ): bupropion [150-300 $\mathrm{mg}$ (mean, $231.8 \mathrm{mg}$ ), $\mathrm{n}=11$ ], aripiprazole [2-5 mg (mean, $3.17 \mathrm{mg}$ ), $\mathrm{n}=3$ ], venlafaxine (102.5 $\mathrm{mg}, \mathrm{n}=1$ ), mirtazapine (22.5 mg, $\mathrm{n}=1$ ), quetiapine (200 mg, $\mathrm{n}=1$ ), amisulpride ( $50 \mathrm{mg}, \mathrm{n}=1)$, risperidone $(2 \mathrm{mg}, \mathrm{n}=1)$, lamotrigine $(25 \mathrm{mg}, \mathrm{n}=1)$. The sedative drugs prescribed with antidepressant treatment at night during the 8 -week of the treatment were as follows ( $\mathrm{n}=55$ ): trazodone [12.5-50 $\mathrm{mg}$ (mean, $32.9 \mathrm{mg}$ ), $\mathrm{n}=30$ ], zolpidem [6.25-12.5 mg (mean, $9.4 \mathrm{mg}$ ), $\mathrm{n}=8$ ], quetiapine [25$200 \mathrm{mg}$ (mean, $58.3 \mathrm{mg}$ ), $\mathrm{n}=6$ ], clonazepam [0.375-0.5 mg (mean, $0.47 \mathrm{mg}$ ), $\mathrm{n}=4$ ], lorazepam [0.5 mg (mean, $0.5 \mathrm{mg}$ ), $\mathrm{n}=4$ ], mirtazapine (22.5 mg, $\mathrm{n}=1$ ), melatonin ( $\mathrm{mg}, \mathrm{n}=1)$, amitriptyline ( $5 \mathrm{mg}, \mathrm{n}=1$ ). The information about the numbers of the combination/augmentation treatment and sedative drugs in the morning and evening/night groups were shown in Table 1.

\section{Depressive symptoms evaluation}

The board-certified psychiatrists assessed the severity of depressive symptoms of all patients using the 17-item Hamilton Depression Rating Scale (HDRS) at the baseline and 8-week after the antidepressant treatment [9]. The treatment response of the 8-week antidepressant treatment was defined as $50 \%$ or more reduction of the HDRS score at 8-week compared to the baseline. The severity of insomnia was assessed by the sum of scores of the item 4 (difficulty in sleep initiation), item 5 (difficulty in sleep maintenance), and item 6 (early morning awakening) of the HDRS. The duration of illness was assessed using the life chart method.

\section{Statistical analyses}

The association between the escitalopram dosing time (i.e., morning vs. evening/night group) and treatment response was analyzed using the logistic regression analysis including age, sex, duration of illness (months), dosage of escitalopram (mg), the number of combination/augmentation (coded as $0-2$ ), and the number of sedative drugs (coded as $0-2$ ) as covariates. For the investigation of the difference of the reduction (\%) of total HDRS score and insomnia-related subscale score after the 8-week of antidepressant treatment between the morning and evening/ night groups, an analysis of covariance (ANCOVA) was performed using the same covariates. The independent $t$-test was used to compare age, duration of illness, HDRS score (baseline, 8 week), and dosage of escitalopram (mg) between two groups. The chi-squared test was used to analyze differences in the distri- 
bution of sex, education level, treatment response, number of combination/augmentation treatment, and number of sedative drugs at night between two groups. All statistical analyses were performed using SPSS Statistics for Windows (version 24.0; IBM Corp., Armonk, NY, USA).

\section{RESULTS}

Demographic and clinical characteristics

Fifty-three patients with MDD were determined as the Morning group, while, eighteen patients were determined as the Evening/Night group in the present sample according to their escitalopram dosing time (Table 1). We could not find any significant difference between two groups regarding age, sex, education level, illness duration, HDRS scores at the baseline and 8 weeks, treatment response, the numbers of combination/augmentation treatment or those with sedative drugs at night, and dosage (mg) of escitalopram (all, p>0.05) (Table 1). The detailed information about the demographic and clinical characteristics of the sample are presented in Table 1.

Association of escitalopram dosing time with response after 8-week escitalopram treatment

As a main analysis, we investigated the association between the escitalopram dosing time and treatment response after 8-week escitalopram treatment. In the analysis, patients in the Morning group did not show any significant difference in likelihood to achieve treatment response at the 8 weeks compared to those in the Evening/Night group (adjusted odds ratio=1.458, $95 \%$ confidence interval $=0.485-4.643, \mathrm{p}>0.1$ ) (Table 2 ).

Difference of HDRS-17 score reduction during 8-week escitalopram treatment between Morning and Evening/ Night dosing group

We also compared the reduction percentage of total HDRS score and insomnia-related subscale score in HDRS after the 8 -week treatment between two groups using the ANCOVA. The reduction percentage was calculated as follows: (score at baseline-score at 8 weeks $) /($ score at baseline $) \times 100$. In the analysis, we could not find any significant difference in the reduction of the total HDRS score (Morning=37.01 $\pm 31.32 \%$, Evening/Night $=46.66 \pm 23.26 \%, \mathrm{p}>0.1$ ) (Table 3 ) and insomnia-related subscale score (Morning=23.18 $\pm 133.25 \%$, Evening/Night $=37.41 \pm 56.78 \%$, p $>0.1$ ) (Table 3).

\section{DISCUSSION}

In the present study, we explored the association between escitalopram dosing time (morning vs. evening or night) and treatment response after 8-week of escitalopram treatment using the sample of patients with MDD in a naturalistic setting. However, we could not find any association between them. We also could not find any significant difference of the changes in global depressive symptoms (i.e., total HDRS score) and insomnia severity (i.e., insomnia-related subscale score) between the Morning and Evening/Night group.

Table 1. Demographic and clinical characteristics of the sample

\begin{tabular}{|c|c|c|c|}
\hline Characteristics & Morning $(\mathrm{n}=53)$ & Evening/Night $(\mathrm{n}=18)$ & $\mathrm{p}$-value $\left(\mathrm{t}, \chi^{2}\right)$ \\
\hline Age (yr) & $40.08 \pm 12.87$ & $42.83 \pm 13.28$ & $0.439(\mathrm{t}=-0.779)$ \\
\hline Sex (female/male) & $38 / 15$ & $12 / 6$ & $0.686\left(\chi^{2}=0.163\right)$ \\
\hline Education level & & & $0.769\left(\chi^{2}=0.526\right)$ \\
\hline Elementary and middle school & 8 & 4 & \\
\hline High school or college/university & 41 & 13 & \\
\hline Above graduate school & 4 & 1 & \\
\hline Duration of illness (months) & $29.53 \pm 34.10$ & $43.89 \pm 96.09$ & $0.352(\mathrm{t}=-0.938)$ \\
\hline HDRS-17 score (baseline) & $18.70 \pm 5.15$ & $18.61 \pm 3.70$ & $0.948(\mathrm{t}=0.066)$ \\
\hline HDRS-17 score (8 wk) & $11.36 \pm 6.06$ & $9.72 \pm 4.25$ & $0.294(\mathrm{t}=1.058)$ \\
\hline Responder/Non-responder & $18 / 35$ & $8 / 10$ & $0.425\left(\chi^{2}=0.636\right)$ \\
\hline No. of combination or augmentation drugs & & & $0.070\left(\chi^{2}=5.327\right)$ \\
\hline 0 & 39 & 15 & \\
\hline 1 & 13 & 1 & \\
\hline (5) & 1 & 2 & \\
\hline No. of sedative drugs at night & & & $0.999\left(\chi^{2}=0.002\right)$ \\
\hline 0 & 15 & 5 & \\
\hline 1 & 35 & 12 & \\
\hline 2 & 3 & 1 & \\
\hline Escitalopram dosage (mg) & $18.40 \pm 4.36$ & $17.50 \pm 3.93$ & $0.443(\mathrm{t}=0.771)$ \\
\hline
\end{tabular}

Data are expressed as mean \pm SD. Combination/augmentation drugs: bupropion, aripiprazole, venlafaxine, mirtazapine, quetiapine, amisulpride, risperidone, lamotrigine. Sedative drugs at night: trazodone, zolpidem, quetiapine, clonazepam, lorazepam, mirtazapine, melatonin, amitriptyline. HDRS-17: the 17-item Hamilton Depression Rating Scale 
Table 2. Association of escitalopram dosing time with response after 8-week escitalopram treatment

\begin{tabular}{lccc}
\multirow{2}{*}{\multicolumn{1}{c}{ Dosing time }} & \multicolumn{3}{c}{ Response } \\
\cline { 2 - 4 } Evening/night & $\mathrm{aOR}$ & $95 \% \mathrm{CI}$ & $\mathrm{p}$-value \\
Morning & 1.458 & $0.485-4.643$ & 0.524 \\
\hline
\end{tabular}

Logistic regression analysis was performed including age, sex, duration of illness (months), dosage of escitalopram (mg), combination/augmentation, and sedative drugs as covariates. Combination/augmentation: number of combination or augmentation drugs on escitalopram treatment (coded as 0-2). Sedative drugs: number of sedative drugs prescribed at night (coded as $0-2$ ). aOR: adjusted odds ratio, CI: confidence interval

Table 3. Difference of HDRS-17 score and insomnia-related subscale score reduction during 8-week escitalopram treatment between Morning and Evening/Night dosing group

\begin{tabular}{|c|c|c|c|}
\hline Score reduction & $\begin{array}{c}\text { Morning } \\
(\mathrm{n}=53)\end{array}$ & $\begin{array}{l}\text { Evening/Night } \\
\quad(\mathrm{n}=18)\end{array}$ & $\begin{array}{l}\mathrm{p} \text {-value } \\
(\mathrm{F})\end{array}$ \\
\hline $\begin{array}{l}\text { HDRS-17 score } \\
\text { reduction (\%) }\end{array}$ & $37.01 \pm 31.32$ & $46.66 \pm 23.26$ & $\begin{array}{c}0.240 \\
(\mathrm{~F}=1.406)\end{array}$ \\
\hline $\begin{array}{l}\text { Insomnia-related subscale } \\
\text { score reduction (\%) }\end{array}$ & $23.18 \pm 133.25$ & $37.41 \pm 56.78$ & $\begin{array}{c}0.653 \\
(\mathrm{~F}=0.204)\end{array}$ \\
\hline
\end{tabular}

Analysis of covariance was performed including age, sex, duration of illness (months), dosage of escitalopram (mg), combination/augmentation, and sedative drugs as covariates. Combination/augmentation: number of combination or augmentation drugs on escitalopram treatment (coded as 0-2). Sedative drugs: number of sedative drugs prescribed at night (coded as 0-2). HDRS-17: the 17-item Hamilton Depression Rating Scale

Escitalopram is the most recently developed selective serotonin reuptake inhibitor (SSRI) and is used widely for the treatment of patients with MDD [10]. The monoamine hypothesis of depression explains the cause and pathophysiology of depression as an altered function of monoamine neurotransmitters in the brain [11]. Consistent with this hypothesis, people who are vulnerable to depression are known to have a weak serotonin system [12]. Such evidences suggested that increased turn-over of serotonin in depressed patients resulted in depletion of serotonin within synapses, resulting in upregulation or down regulation of 5-HT receptors [13]. SSRIs administration is assumed to modulate the altered serotonin system and it can lead to improvement in depressive symptoms.

At the level of neurotransmission, the serotonin system has attracted particular interest in relation to the regulation of circadian rhythms [14]. Serotonin is an indoleamine known as the precursor to melatonin. In rodents, brain serotonin levels change across the day, entrained to light onset and offset $[15,16]$. Early post-mortem studies have demonstrated a marked seasonal as well as circadian variation of serotonin concentration in several brain regions [17]. Moreover, molecular imaging studies have reported seasonal changes in the serotonin system [18].

Evidence suggests that failing to achieve full remission in depression significantly impairs patient functioning and increases the risk of relapse [19]. Since depression is among the most disabling and costly disorders, achieving and sustaining remission is of particular concern to clinicians [20]. Therefore, it is important for patients suffering from depression not just to have their symptoms improved but also to achieve genuine well-being. Although we did not find any association between dosing time of antidepressant and treatment response, dosing time is a crucial aspect of precision medicine which aims to find the right drug for the right patient at the right dose. However, the discussion regarding precision medicine is almost entirely focused on genetic precision until now. Because several lines of evidence suggest that serotonin system has the circadian rhythm as described above, dosing time may play a role in the therapeutic effects of antidepressant.

There are some limitations in the present study. Firstly, the relatively small sample size limits the generalizability of our results. The data presented above need to be confirmed with a much larger sample size. Secondly, there was a significant difference in the concomitant drugs. They were included as a covariate (coded as $0-2$ ) when analyzing the association. However, differences in these concomitant drugs, especially sedatives, may have an effect on the result. Combination or antianxiety medication could compensate the insufficient effect of antidepressant. Further studies with larger samples and controlling the effect of concomitant medication are therefore warranted.

In conclusion, although we did not find any significant association between escitalopram dosing time and treatment response, there is no doubt about an importance of timely dosing. Further researches with larger size sample and various antidepressants are needed.

\section{Acknowledgments}

None

\section{Conflicts of Interest}

The authors have no potential conflicts of interest to disclose.

\section{Author Contributions}

Conceptualization: Ho-Kyoung Yoon. Data curation: Kyu-Man Han. Formal analysis: Kyu-Man Han. Methodology: Ho-Kyoung Yoon, Kyu-Man Han. Writing_-original draft: Ho-Kyoung Yoon, Kyu-Man Han. Writing-review \& editing: Ho-Kyoung Yoon.

\section{ORCID iDs}

Ho-Kyoung Yoon (1)

https://orcid.org/0000-0002-3302-0660

Kyu-Man Han (1)

https://orcid.org/0000-0002-1982-4216

\section{REFERENCES}

1. Klevecz RR, Shymko RM, Blumenfeld D, Braly PS. Circadian gating of S phase in human ovarian cancer. Cancer Res 1987;47:6267-6271.

2. Levi FA, Hrushesky WJ, Blomquist CH, Lakatua DJ, Haus E, Halberg F, et al. Reduction of cis-diamminedichloroplatinum nephrotoxicity in rats by optimal circadian drug timing. Cancer Res 1982;42:950-955.

3. Lévi F. From circadian rhythms to cancer chronotherapeutics. Chronobiol Int 2002;19:1-19. 
4. Ancoli-Israel S, Liu L, Marler MR, Parker BA, Jones V, Sadler GR, et al. Fatigue, sleep, and circadian rhythms prior to chemotherapy for breast cancer. Support Care Cancer 2006;14:201-209.

5. Xu J, Winkler J, Sabarinath SN, Derendorf H. Assessment of the impact of dosing time on the pharmacokinetics/pharmacodynamics of prednisolone. AAPS J 2008;10:331-341.

6. Muller JE, Stone PH, Turi ZG, Rutherford JD, Czeisler CA, Parker C, et al. Circadian variation in the frequency of onset of acute myocardial infarction. N Engl J Med 1985;313:1315-1322.

7. Edwards PA, Muroya H, Gould RG. In vivo demonstration of the circadian thythm of cholesterol biosynthesis in the liver and intestine of the rat. J Lipid Res 1972;13:396-401.

8. Knopp RH. Drug treatment of lipid disorders. N Engl J Med 1999;341:498511.

9. Hamilton M. A rating scale for depression. J Neurol Neurosurg Psychiatry 1960;23:56-62.

10. Kennedy SH, Andersen HF, Thase ME. Escitalopram in the treatment of major depressive disorder: a meta-analysis. Curr Med Res Opin 2009;25:161-175.

11. Schildkraut JJ. The catecholamine hypothesis of affective disorders: a review of supporting evidence. Am J Psychiatry 1965;122:509-522.

12. Fakhoury M. Revisiting the serotonin hypothesis: implications for major depressive disorders. Mol Neurobiol 2016;53:2778-2786.

13. Charney DS, Menkes DB, Heninger GR. Receptor sensitivity and the mechanism of action of antidepressant treatment. Implications for the etiology and therapy of depression. Arch Gen Psychiatry 1981;38:1160-1180.

14. Ciarleglio CM, Resuehr HE, McMahon DG. Interactions of the serotonin and circadian systems: nature and nurture in rhythms and blues. Neuroscience 2011;197:8-16.

15. Birkett M, Fite KV. Diurnal variation in serotonin immunoreactivity in the dorsal raphe nucleus. Brain Res 2005;1034:180-184.

16. Cagampang FR, Yamazaki S, Otori Y, Inouye SI. Serotonin in the raphe nuclei: regulation by light and an endogenous pacemaker. Neuroreport 1993;5:49-52.

17. Bucht G, Adolfsson R, Gottfries CG, Roos BE, Winblad B. Distribution of 5-hydroxytryptamine and 5-hydroxyindoleacetic acid in human brain in relation to age, drug influence, agonal status and circadian variation. J Neural Transm 1981;51:185-203.

18. Matheson GJ, Schain M, Almeida R, Lundberg J, Cselényi Z, Borg J, et al. Diurnal and seasonal variation of the brain serotonin system in healthy male subjects. Neuroimage 2015;112:225-231.

19. Keller MB. Past, present, and future directions for defining optimal treatment outcome in depression: remission and beyond. JAMA 2003;289:31523160 .

20. McIntyre RS, O'Donovan C. The human cost of not achieving full remission in depression. Can J Psychiatry 2004;49(3 Suppl 1):10S-16S. 